\title{
Melanosis of the bladder: a case report
}

\section{Introduction}

Melanosis of the bladder is a rare entity with only a few cases reported in the literature to date. The exact incidence is unknown. It remains under debate whether melanosis of the bladder is a benign condition. Although it is generally considered benign; it has also been associated with malignant melanoma and transitional cell carcinoma. Therefore it is important to take biopsies of the pigmented urothelium to prevent misdiagnosis. We report a patient with pigmentation of the bladder mucosa diagnosed as melanosis vesica.

\section{Case report}

A 47-year-old woman was referred to our Department of Urology with abdominal pain, urgency and urinary incontinence. The urine sediment showed microscopic hematuria and urine culture was negative. Patient's past medical history is positive for recurrent urinary tract infections and two caesarean sections. CT-scan showed no evidence of disease. Cystoscopy revealed black-pigmented urothelium on the posterior wall of the bladder (Figure 1). Urine cytology showed normal urothelial cells with pigmentation. Cold cup biopsies of the pigmented urothelium demonstrated normal urothelial cells with black pigments focally present in the cytoplasm. There were no melanocytes identified in Hematoxylin and Eosin stained sections (Figure 2), or in the additional immune histochemical stainings for melanocytic markers (S-100, Melan-A, SOX10 and HMB-45). Special histochemical staining was performed; the Schmorl staining (Figure 3) and Fontana Masson for detecting melanin were positive. Periodic Acid Schiff for detecting lipofuscin and Perls stain for detecting hemosiderine were negative. The CD68 staining showed macrophages in the superficial lamina propria with melanin pigment in the cytoplasm. Also loose melanin pigment deposits could be appreciated between these macrophages. These cystoscopic and histopathologic findings were diagnostic for bladder pigmentation due to melanosis vesica.

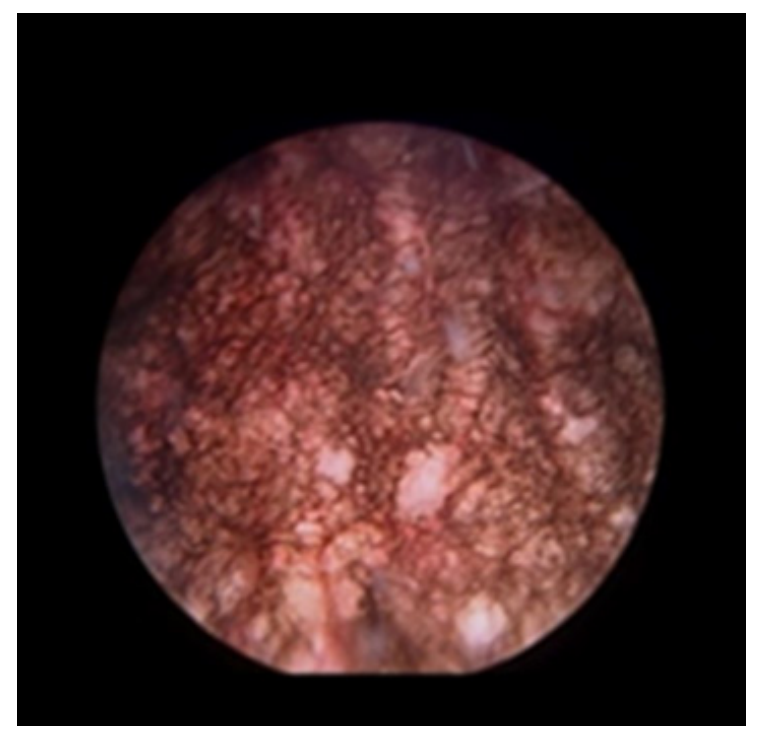

Figure I wall of the bladder.

\section{Zietse CS,' Licht JR, ${ }^{2}$ Stuurman Wieringa RE' 'Department of Urology, Reinier de Graaf Gasthuis Delft, the Netherlands \\ ${ }^{2}$ Department of Pathology, Reinier de Graaf Gasthuis Delft, the Netherlands}

Correspondence: Zietse CS, Department of Urology, Reinier de Graaf Gasthuis, Reinier de Graafweg 3-I I0, 2625 AD Delft, The Netherlands, Email carlijnzietse@gmail.com

Received: August 06, 2017 | Published: June 21, 2017

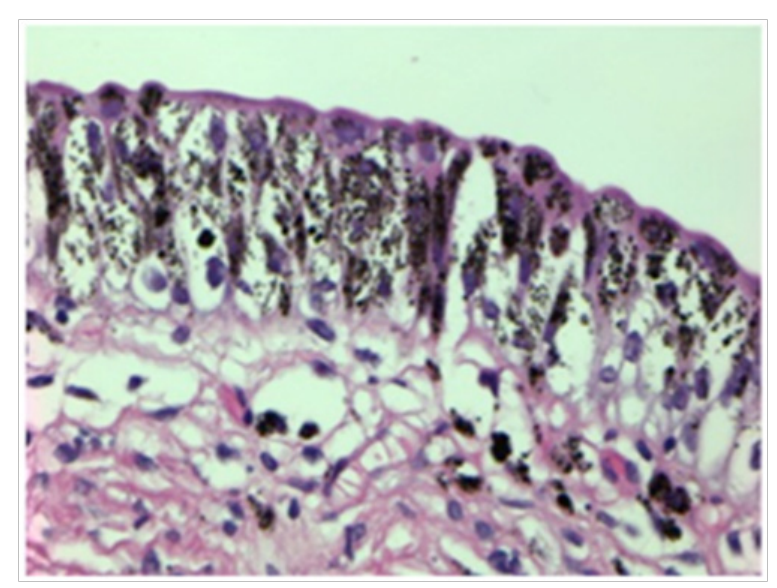

Figure 2 Eosin stained sections.

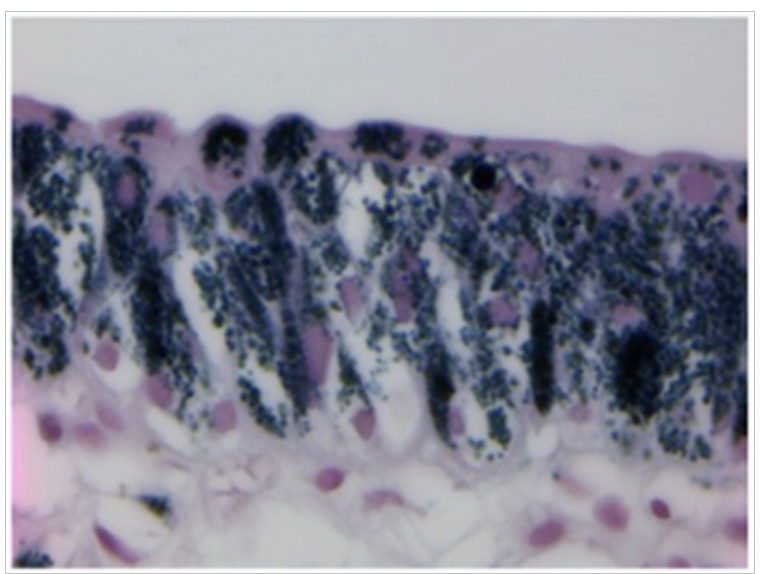

Figure 3 Schmorl staining. 


\section{Discussion}

Melanosis is a descriptive term used for dark brown-black tissue pigmentation due to melanine deposition. ${ }^{1}$ Melanosis has been seen in several other organs such as the skin, colon and oral mucosa. ${ }^{2}$ Melanosis vesica, deposition of melanin pigment in the urothelium of the bladder is a rare condition. The exact incidence of this condition is unknown with only a few cases reported in the literature to date. ${ }^{3-6}$ Patients presented with different urinary tract symptoms such as haematuria, dysuria, incontinence and urinary tract infections. ${ }^{3}$ It remains unclear whether symptoms were related to the depositions of pigment in the bladder. On cystoscopy, the mucosa of the bladder shows a brownblack discoloration. The discoloration can be present in a spotted pattern or diffuse in all the quadrants of the bladder. Microscopic examination reveals brown-pigmented granules within the urothelial cells and sometimes the lamina propria. Biopsies must be taken to rule out serious pathology. The differential diagnosis of pigmentation of the bladder urothelium is hemosiderosis, lipofuscinosis, melanosis and primary or metastatic melanoma. These can be distinguished by histopathological examination.

Melanosis vesica is considered a benign condition in the literature. Melanosis is used interchangeably to describe bladder pigmentation by melanin with presence or absence of melanocytes. The bladder mucosa normally does not contain melanocytes. An association between the presence of melanosis in the bladder mucosa and malignant melanoma is described. Kerley at al. ${ }^{7}$ reported a case of a 80 -year old women with malignant melanoma of the labia majora and melanosis of the bladder. She underwent a vulvectomy. Three years later she presented with a multifocal malignant melanoma of the bladder and vagina. It remains unclear whether melanocytes were present in the urothelium when the diagnosis melanosis was made. Two other cases were reported transitional cell carcinoma in association with melanosis of the bladder. Both cases show no melanocytes in the urothelium. Sanborn et al. ${ }^{3}$ described a case of a 63 -year old women with melanosis of the bladder who one year later developed a transitional cell bladder carcinoma. Harikrishnan et al. ${ }^{5}$ report a case of a 50 -year-old man who presented with melanosis vesica described as a diffusely pigmented bladder, without the presence of melanocytes. Besides the melanosis there was a renal and distal right ureter transitional cell carcinoma at presentation. An association is assumed but it remains unclear whether melanosis of the bladder and the malignancies in these patients were related. Therefore it still remains under debate whether melanosis of the bladder is a benign condition. Follow up by regular cystoscopic examination is recommended.

\section{Acknowledgments}

None.

\section{Conflicts of Interset}

None.

\section{References}

1. Stuhldreher PP, Ponsky LE, MacLennan GT. Melanosis of the bladder. $J$ Urol. 2011;185(5):1908-1909.

2. Talmon GA, Khan AU, Koerber RK, et al. Simple melanosis of the bladder: a rare entity. Int J Surg Pathol. 2010;18(6):547-549.

3. Sanborn L, MacLennan G, Cooney MM, et al. High-grade transitional cell carcinoma and melanosis of the urinary bladder: case report and review of the literature. Urology. 2009;73(4): \928.e13-928.e15.

4. Jin B, Zaidi SY, Hollowell M, et al. A unique case of urinary bladder simple melanosis: a case report and review of the literature. Diagn Pathol. 2009;4:24

5. Harikrishnan JA, Chilka S, Jain S. Urinary tract transitional cell carcinoma and melanosis of the bladder: a case report and review of the literature. Ann R Coll Surg. 2002;Engl 94:e152-e154.

6. Smeets MHJ, de Wit PEJ, van der Schoot DKE. Case Report. (Pseudo)melanosis van de blaas. Tijdschrift voor de urologie juni. 2003;3(4):111-116.

7. Kerley SW, Blute ML, Keeney GL. Multifocal malignant melanoma arising in vesicovaginal melanosis. Arch Pathol Lab Med. 1991;115(9):950-952. 